\title{
MODELS AND METHODS OF SUPPORT DECISION FOR MANAGEMENT OF IT COMPANIES
}

\author{
Yehor Tatarchenko ${ }^{1}$ \\ gosahi@gmail.com
}

Volodymyr Lyfar ${ }^{1}$

lyfarva61@ukr.net

\author{
${ }^{1}$ Department of Programming and Mathematics \\ Volodymyr Dahl East Ukrainian National University \\ 59-a Tsentralnyi ave., Severodonetsk, Ukraine, 93400
}

\begin{abstract}
Studies have been carried out and methods have been proposed to increase the competitiveness of IT companies by improving the quality of their state analysis taking into account stochastic indicators. Growth conditions may provide mergers and acquisitions (M\&A). One of the important components of mergers and acquisitions is a qualitative assessment of the value and condition of the company associated with the development of IT. Particularly tangible are the results of a qualitative financial analysis for IT companies whose activities are aimed at the distribution and support of energy resources in a competitive environment of generating and supplying energy companies in the regions. The analysis of the data obtained as a result of the P\&L financial report is mainly based on current indicators and can be partially used to prolong economic indicators for a certain (most often limited) period. In this case, the stochastic characteristics of non-interconnected influencing processes are practically not determined and quantitatively not taken into account. Thus, the definition of qualitative indicators of the economic state is most often based on a balanced scorecard (Balanced Scorecard, BSC). The authors propose using methods for evaluating stochastic indicators of IT development processes based on a number of tasks:

1) development and coordination of methods and models that allow for the assessment of influencing indicators in the analysis of the financial condition of the analyzed companies, taking into account the likelihood of the implementation of scenarios of their development;

2) creation of an information model and methods for processing current stochastic data and assessing the probability of the implementation of negative and positive outcomes.
\end{abstract}

Keywords: IT project, project management, risk, decision-making, mergers and acquisitions, failure trees, event trees.

DOI: $10.21303 / 2461-4262.2020 .001363$

\section{Introduction}

The development of information technology projects includes procedures based on a special project-driven approach to all stages of the project life cycle [1]. At the same time, such projects are among the most risky investments [2]. According to the data of Standish Group [3], 31.1\% of all projects in the industry will remain incomplete, $52.7 \%$ will have a cost that is almost 2 times higher than the declared value. In general, the probability of successful implementation and implementation (maintenance) of an IT project is less than 0.5. That is, the economic indicators of enterprises that fulfill orders in the IT sector in the face of fierce competition can't ensure the existence of enterprises with such risk indicators.

Ukraine ranked 73rd out of 166 countries in 2014 according to the International Telecommunication Union at the UN in the field of information technology and 79th in 2017, catching up with Uzbekistan and Kazakhstan [4]. The same place outside Ukraine remained in 2018, which indicates a trend of deterioration in this area. Despite this, the economic attractiveness of IT developments is explained by high investment activity and the possibility of making a profit without significant financial investments in tangible objects (real estate, materials, raw materials, etc.) [5].

The practical decrease in the competitiveness of IT companies is partly due to the weak level of support for managing risks arising from the implementation and implementation of IT projects, as a reaction to market factors that are not amenable to control by the management [6]. The current business climate is characterized by continuous competition, profit-shifting and rapidly changing 
technologies. Moreover, the instability in the market of IT services is often caused by a random cause independent of the enterprise management [7]. For example, the Ukrainian rapidly growing market of IT services is characterized by instability of financial indicators and the difficulty of maintaining the proper level of competitiveness of IT companies. The use of mergers and acquisitions (M\&A) to manage the market of services and production in the field of information technology can significantly stabilize the risks of financial investments in the IT market [8]. One of the important components of mergers and acquisitions is a qualitative assessment of the value and condition of the company associated with the development of IT. The main objective of mergers and acquisitions is to increase the value of the objects created by the company. At the same time, the cost of combining firms should become greater than the total values of the merger component. Violation of this rule is possible if the risk assessment of the state of companies is incorrect. Of course, first of all, from the indicators of the "value" of the company, it is necessary to determine its financial value and solvency. In this case, it is necessary to analyze simultaneously indicators that affect the financial condition. It is necessary to try to achieve the highest possible synergies (additional value arising as a result of M\&A) [9].

The solution of these problems is associated with the use of a more complex methodological database for data processing, a wide range of variables, the use of various models and algorithms for assessing values and costs [7].

However, even in this case, obtaining reliable estimates in the analysis of the data, provided that there are a large number of different factors, may be questioned in connection with the stochastic nature of the input data. It is in connection with the above that a number of important scientific and technical problems can be distinguished that can be solved by combined methods of mergers and acquisitions and methods of assessing the probability of occurrence of consequences from mergers and acquisitions.

\section{Models, methods and tasks of researching indicators of the state of IT companies}

Obtaining indicators of the state of companies uses a variety of methods, such as: DCF analysis, comparability analysis of companies, assessment of case studies [9]. Most methods and operations are based on the analysis of economic and, partially, technological indicators. But it is also important to take into account the stochastic characteristics of the studied objects and apply methods and models to assess the likelihood and consequences of changes in these indicators. Thus, decision-making regarding the status and management of IT projects can be based on priority methods and models of risk assessment stages of IT development [10].

Models and methods of financial estimates are widely represented and implemented by software products [8], such as:

- package for modeling business valuation;

- FP\&A Monthly Cash Flow Forecast Modeling;

- package of scripts and sensitivity analysis;

- financial model of a startup;

- modeling of mergers and acquisitions (M\&A);

- LBO Model - Leveraged Buyout Modeling Course;

- NPV ("net present value");

- ROI ("return on investment").

However, there are no full-scale methods for assessing P\&L (presented mainly in the financial statements of companies) generally accepted for evaluating IT companies [9]. In addition, the dynamic factor of the impact of the state of development of IT projects on income and expenses is not fully taken into account.

Analysis of the data obtained as a result of the P\&L financial report most often relies on current indicators and can be partially used to prolong economic indicators for a certain (most often limited) period [9].

In this case, the stochastic characteristics of unrelated influencing processes are practically not determined and quantitatively not taken into account [11]. For example, such as the qualification level of the development team, their level of motivation, the regulatory framework that determines the work of IT companies and many others [9]. 
The main feature of using probabilistic models is that the financial consequences of developing an IT project are considered as a random variable. Project risks are taken into account in the features of the distribution functions of stochastic indicators [11]. In this sense, the concept of "risk" is defined as the probability of the implementation of certain events under certain conditions in a given period of time. Stochastic events themselves must be formalized and can be evaluated both qualitatively and quantitatively on the basis of criteria parameters characteristic of the consequences of their implementation.

Moreover, the definition of qualitative indicators of the economic state is most often based on a balanced scorecard (Balanced Scorecard, BSC) [12].

Thus, the problem of high-quality decision-making regarding the activities of the analyzed IT companies can be based on solving a number of problems in assessing the state of financial flows and competitive indicators (Fig. 1).

In particular, all the indicators of financial flows discussed above can be estimated in the future by BSC indicators [9], for example:

- use value - by parameters of the quality of products and services, as well as characteristics of the impact on customers and consumers;

- competitiveness - by characteristics of market parity;

- technological indicators - comparison of the package of technologies used with the most advanced innovations (advanced technologies, certification tools, recognized leaders); of formalization).

- competency level - share of certified employees and business processes (a certain level

A high-quality informational assessment is carried out on the basis of the assessment of changes in BSC indicators, the calculation of the total indicators of the weights of various indicators and the search for projects with the maximum total category by comparing the groups of indicators.

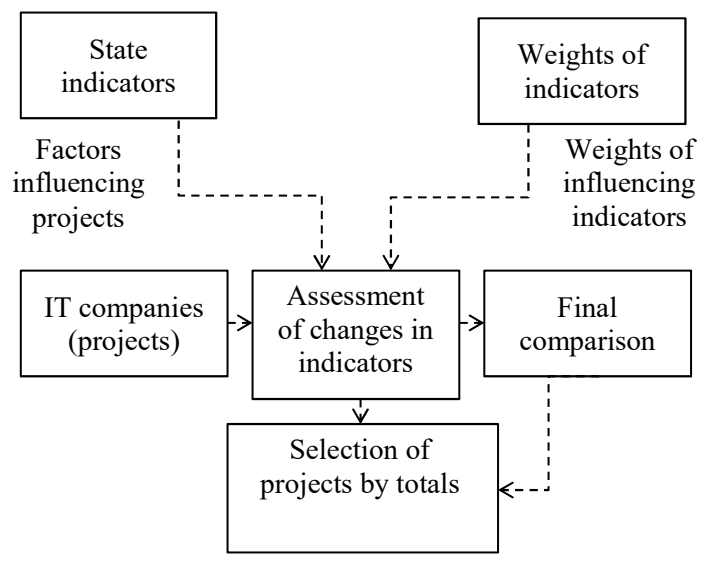

Fig. 1. High-quality informational assessment of IT project

All presented indicators can be estimated and ranked according to the degree of their deviations and the probability of reaching certain values (both positive and negative in the economic sense).

Research objectives are as follows:

- it is necessary to develop and coordinate methods and models that allow, in a given confidence interval, to assess the financial, technological, organizational and other influential indicators of the analyzed companies and the likelihood of their implementation;

- develop methods for processing current stochastic data and assess the likelihood of the implementation of negative and positive outcomes of the forecasted conditions of the analyzed companies;

- develop methods and a decision support system in the face of uncertainty, implemented in information technology to ensure the management of the activities of the analyzed IT companies. 


\section{Implementation of scenarios for the development of the state of IT companies}

To solve this problem, an approach has been taken to make optimal decisions in the Pareto sense based on the probability of implementing scenarios for the development of the state of IT companies in conflict and uncertainty. By "conflict" is meant the competitive development of any indicators (positive and negative, profit and loss) of the current state of the analyzed objects.

In this case, the risk is considered as the occurrence of certain events with a certain probability. Moreover, all events can be characterized by quantitative (for example, value) or qualitative (for example, permissible, unacceptable) indicators.

Using the concepts of game theory, the state of the analyzed objects is presented as a matrix of probable states obtained on the basis of event trees (ET). Moreover, each strategy $\mathrm{S}_{\mathrm{j}}$ represented by the proposed or proposed set of impacts on an object is evaluated either quantitatively through indicators of profitability or losses, or qualitatively through characteristic indicators of levels of positive or negative outcomes. Moreover, mixing estimates is unacceptable.

The generalized matrix of the matrix of probable states can be represented as follows:

$$
\begin{aligned}
& \begin{array}{llll}
S_{1} & S_{2} & . . & S_{n}
\end{array} \\
& \begin{array}{c}
P_{1} \\
P_{2} \\
. . \\
P_{m}
\end{array}\left(\begin{array}{cccc}
e_{11} & e_{12} & . . & e_{1 n} \\
e_{21} & e_{22} & . . & e_{2 n} \\
. . & . . & . . & . . \\
e_{m 1} & e_{m 2} & . . & e_{m n}
\end{array}\right) \text {, }
\end{aligned}
$$

where $P_{i}$ - the set of probabilities of the resulting events $e_{i j}$. To each final event $e_{i j} \rightarrow p_{i j} ; p_{i j} \in P_{i}$. Thus, the task of state analysis is based on a set of methods that allow to model and evaluate the effects of influencing influences (determine the consequences of $e_{i j}$ events) and put in them a surjective map of the set of probabilities of these consequences.

The consequences of the analyzed events are estimated using the models described above or individual models for which formalization is possible for known deterministic models of processes that may occur in the $i$-th subsystem when determining the status of companies: $e_{i j} \rightarrow p_{i j} ; p_{i j} \in P_{i}-$ (a set of elementary events), where $\vec{S}_{i j}$ - a vector of parameters that determines the initial state of the $i$-th subsystem; $\vec{\Phi}_{i j}$ - a vector of phase variables of elementary actions that can occur in the $i$-th subsystem under such actions.

To determine the quantitative probability values of initial events that determine the initial state of the subsystem, FTA analysis methods (fault trees) are implemented, and an upward analysis of ETA event trees is used to determine the development of scenarios of probable outcomes.

\section{Methods of risk management of IT developments}

The logical sequence of actions that implement the proposed method is illustrated in Fig. 2.

The set of initial events in the FTA is determined by an expert (group of experts) as a result of applying a formalized analysis of the conditions of IT companies based on preference methods or ranking assessments [11]. At the same time, it is important that the set of selected initiating events contain a limited set of conditions that are most significant in terms of critical parameters and could significantly affect the state of the IT companies.

Fig. 2 demonstrates a method for analyzing the possible states of an IT company (or project). All information processing processes are divided into three "areas". Failure Tree Construction Area (FTA). In it, a top-down analysis establishes the causal logical relationships of failures or prohibitions of the implementation of source events, which allow to calculate the probabilities of the implementation of the "upper events" $P_{i}$. Next, each top event with a calculated probability of its implementation is mapped to the area of construction of "event trees" (ETA). In it, an upward analysis establishes a connection between the current state and some $V_{i}$ action, which introduces binary branching with the probability of the implementation of this effect or denial of implementation. Moreover, the probability of the impact can also be defined as some "upper event" of the corresponding fault tree. The result of the construction of such trees are finite branches, weighted by the $\Phi_{i} ; M_{i} \Phi_{i}$ has a structure of qualitative (semantic) indicators of the state of development 
of the company (project) and quantitative indicators of the probability of achieving such a state. $M_{i}$ - reflects some financial (economically) consequences of the i-th scenario of the development of events. In the "scenario area", each scenario establishes mutual compliance with the decisions taken on mergers and acquisitions and the possible gains and losses of such decisions determined by experts (effect of impacts). This allows to determine the values of the expected damage and expected profit. The results obtained allow to carry out a comparative analysis of the expected conditions and decisions, taking into account economic indicators and the likelihood of their achievement. In Fig. 2, the red dashed line shows the passage combination along tree branches from a certain initial event FTA1 to the scale of consequences M1. Such combinations are "weighted" by quantitative indicators of the probability and cost of the consequences and can be sorted by various criteria. This makes it possible to analyze and search for the most rational solutions.

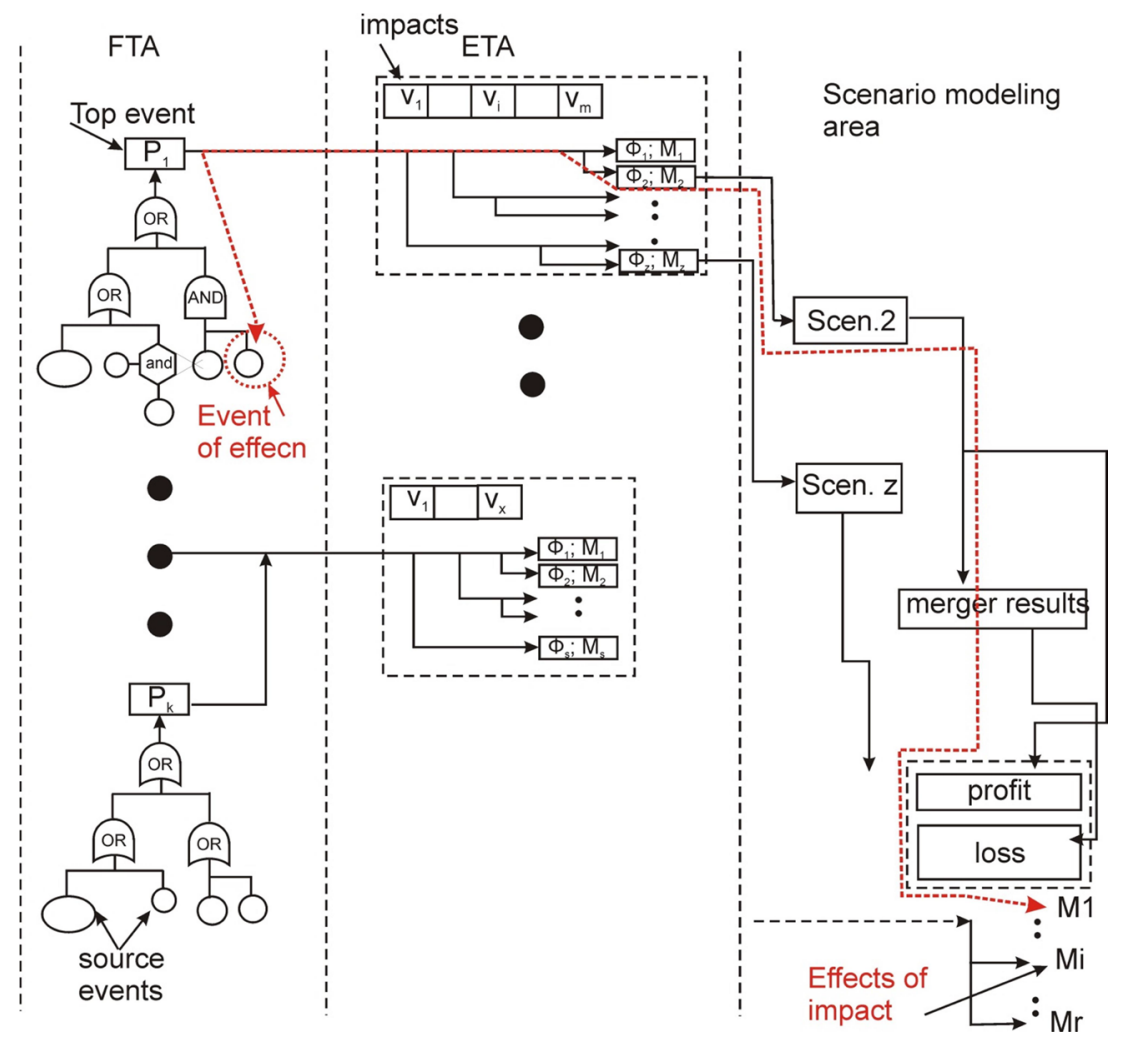

Fig. 2. Illustration of the logical connections of the information model in the decision support system for mergers and acquisitions

The construction of "fault trees" is based on a sequential logical descent of cause-effect relationships between events from the top event (using heuristic rules) through logical operations down to the achievement of "elementary events". The probability values of the lower events are determined deliberately and are usually stored in databases organized and updated by methods determined by the researchers. Failure trees examine stochastic events and processes. The probability of their implementation over time is taken into account by processing the statistical data of the initial events, taking into account the normalization of all processes to the times of the corresponding life cycles of IT developments (Table 1).

When creating a "fault tree" by setting the nodes of the corresponding logical operations for joint and incompatible events, branches are formed that correspond to the probability formula for the implementation of the upper event [14]. In this case, the formula for calculating the probability of the upper event is automatically generated, which is based on elementary events, the probability of which is considered known. Combinations of fault trees make it possible to determine the field of the risk component $P_{i}$. 
Table 1

Probability formulas for logical operations

\begin{tabular}{ccc}
\hline $\mathbf{A N D}(\wedge)$ & $\mathbf{O R}(\mathrm{V})$ & $\mathbf{X O R}(\oplus)$ \\
\hline$P_{e}=\prod_{i=1}^{n} P_{i}$ & $P_{e}=1-\prod_{i=1}^{n}\left(1-P_{i}\right)$ & $P_{e}=\sum_{i=1}^{n} P_{i}$
\end{tabular}

Next, for each corresponding upper FTA event, an event tree (ETA) is constructed by the upward analysis method, the branches of which are determined by influencing factors $V_{j}$. Influencing factors can be both financial and organizational decisions to change the activities of the IT company. For example, financing some innovation or strengthening the staff of programmers.

The resulting reaction of such decisions can be both positive and negative effects (including those without consequences), which are realized with different probability.

Thus, as a result of the analysis of the activities of IT companies, a structural-logical model was built. This model allows to automate the processing of incoming information flows about the activities of IT companies and to monitor the current state of the studied objects in the market of this sphere, which was proved experimentally.

To demonstrate the proposed methods, pilot development studies were conducted for various projects of three IT companies. At the same time, P\&L assessments of the current state of the companies under study were carried out using rank risk assessment methods and methods for quantitatively assessing the likelihood of implementing individual company development scenarios. Trend analysis of the dynamics of financial indicators is carried out on the basis of the data obtained over the course of one year. Research is conducted by independent experts to determine the likelihood of companies developing with a surplus of approximately one level.

As a result of the analysis of the cause-effect relationships of events within the companies, failure trees for each company were built and branches which events maximally affect the probability of the top event of the corresponding FTAs are selected. According to the results of calculations, the probability of a surplus in development by the studied companies was distributed as follows: $1 \mathrm{st}-0.645 ; 2 \mathrm{nd}-0.487$; $3 \mathrm{rd}-0.51$. Obviously, the effectiveness of the 1 st company is the highest. However, a further study of the likelihood of achieving this effect showed that indicators of the reliability of the development of events when the work schedule is required and the implementation is successful give different results for the expected effect. FTA and ETA are built on the basis of expert assessments of three independent experts and a control assessment of probable conditions is obtained. An example of the development of trees and their concatenation for one of the scenarios is presented in Fig. 3. For all studied IT companies, the results of the expected surplus in accordance with the probability of its achievement are:

- for the 1 st $0.647 \cdot 0.21=0.1359$;

- for the 2nd $0.487 \cdot 0.65=0.3165$;

- for the 3 rd $0.51 \cdot 0.63=0.3213$.

The above calculation results confirm that the priority sequence for the takeover is the following sequence: 3-2-1 companies.

Fig. 3 shows an example of constructing logical connections between the initial and intermediate events of the flesh before the stock scenario for the first company under study. Construction and calculations are performed using a specialized software product developed by the authors of the article. A simplified example to facilitate demonstration of the functions of the proposed methods. The FTA is built separately from the ETA, after which concatenation occurs at the top event of the fault tree. The functional of the logical information model allows to select the branches of the combinations of events and analyze the level of influence of the initial events on the probability of reaching the final scenario. In Fig. 3 the branch is highlighted in color, which gives the maximum contribution to the probability of the upper event (in this case, 0.647). 

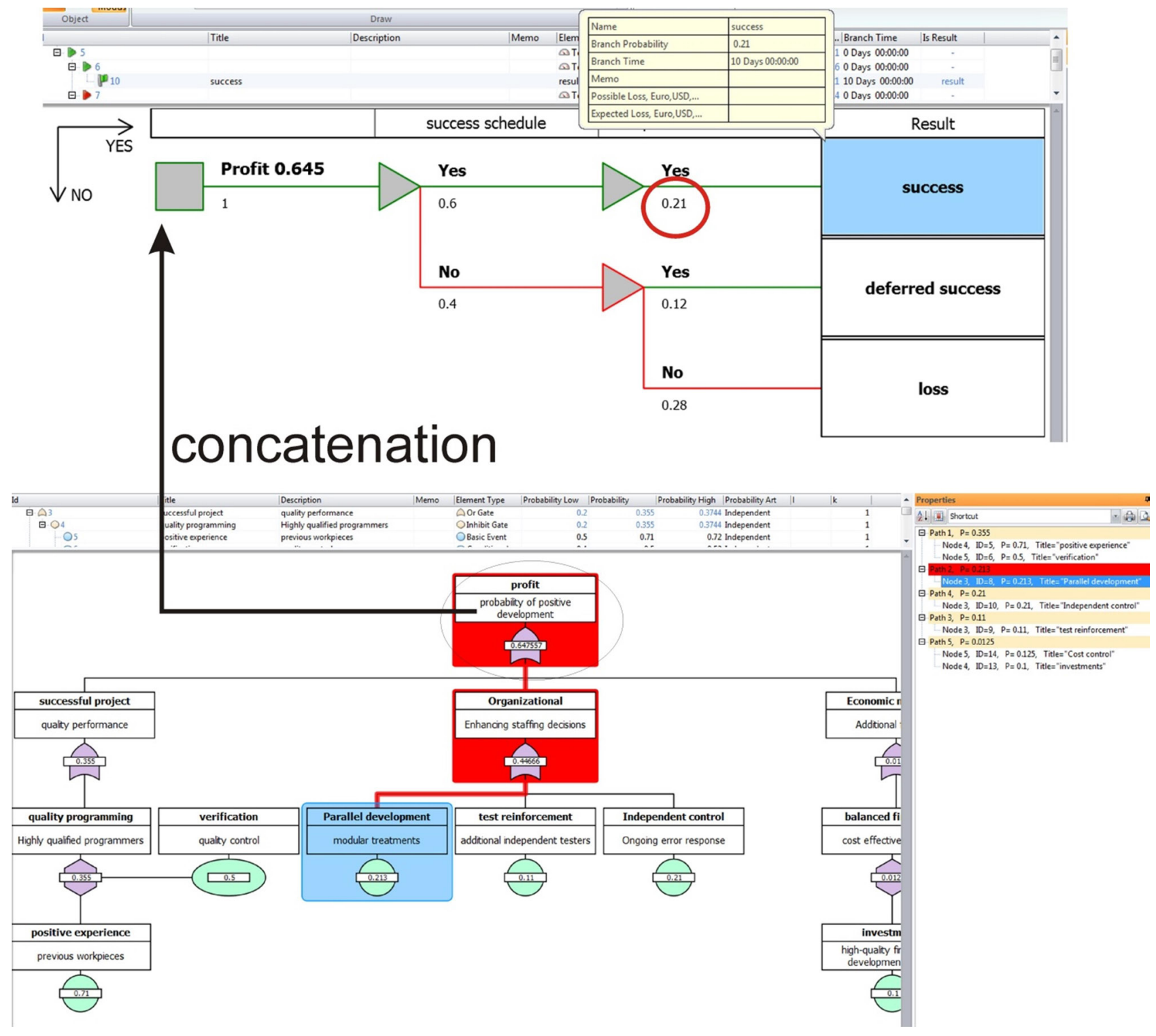

Fig. 3. An example of determining the probability of the implementation of part of the scenarios of potential decisions regarding the implementation of the schedule and project implementation

The given example characterizes priorities in only one parameter - the expected surplus. To perform an analysis using several parameters (multi-parameter optimization), it is necessary to apply more complex navigation methods in the Pareto space. This is due to the fact that, with multi-parameter optimization, alternative solutions are developed and it is necessary to use the mechanisms of graph theory to weight the results of states combining many parameters.

\section{Discussion of research results on methods for analyzing risk components of the stages of IT development}

Each year, the future becomes more uncertain [6] and one of the main factors of this uncertainty is technological development [15]. Of course, risk assessment of the implementation of negative or positive results of the implementation of the adopted organizational, technical and economic decisions on the relativity of the activities of IT companies can be performed by the methods described in [13]. At the same time, IT companies are trying to reduce uncertainty by various approaches [16], which, in principle, speaks of a systematic approach, but not of the accuracy of risk determination [6]. But the methods of quantitative assessment of risk indicators make it possible to 
more accurately determine the level of risk in a narrower confidence interval, in contrast to [17]. In addition, the methods presented make it possible, by creating a database and a knowledge base and using probability theory, to improve the estimates of the probability of certain consequences due to the accumulated experience and analysis of events in the IT market.

Considering $[4,5]$, it is possible to add that the developed information model and software for its implementation are the basis of the decision support system for mergers and acquisitions, as well as the work of IT companies and their strengthening in the information services market. This will not only identify and analyze the risk, as proposed in [12], but also significantly expand the cause-andeffect base of relationships of individual risk indicators that affect the work of IT companies in the market. And this, unlike the one proposed in [1], allows to analyze the occurrence and development of risk during the transition of an IT company between different stages of its life cycle.

\section{Conclusions}

1. As a result of the research, methods are developed and agreed upon to analyze the stochastic and determinate risk components of the effects of events affecting the development life cycle of IT company developments. These methods are based on the construction of an oriented logical causal relationship of the initial and subsequent events and effects. Unlike existing rank expert methods, their application allows to determine the quantitative values of profit and loss and the likelihood of their scenarios.

2. A conceptual approach to solving problems of processing information about the state of IT companies is proposed taking into account the likelihood of the development of positive or negative consequences of decisions. The novelty of this approach lies in the proposed mechanism for the joint use of FTA and ETA, as well as a comparative analysis of the expected magnitude of the consequences. Unlike existing methods of analyzing the financial condition, this method allows to take into account the stochastic characteristics of processes that have a significant impact on the quality of decisions regarding mergers and acquisitions.

\section{References}

[1] Project Management from Simple to Complex (2016). University of Minnesota Libraries Publishing edition. doi: https:// doi.org/10.24926/8668.2101

[2] Kononekno, I. V., Haraziy, A. V. (2012). Razrabotka metoda analiza informatsii dlya vybora optimal'noy metodologii upravleniya proektom. Eastern-European Journal of Enterprise Technologies, 1 (13 (55)), 4-7. Available at: http://journals.uran.ua/ eejet/article/view/3634/3407

[3] The Standish Group Report. Chaos. Available at: https:/www.projectsmart.co.uk/white-papers/chaos-report.pdf

[4] Measuring digital development. Facts and figures. Available at: https://www.itu.int/en/ITU-D/Statistics/Documents/facts/ FactsFigures2019.pdf

[5] Ukraine Country Profile 2019. American Chamber of Commerce in Ukraine. Available at: http://www.chamber.ua/Content/ Documents/629107533Country_Profile_2019_EN.pdf

[6] Mohammed, H. K., Knapkova, A. (2016). The Impact of Total Risk Management on Company’s Performance. Procedia - Social and Behavioral Sciences, 220, 271-277. doi: https://doi.org/10.1016/j.sbspro.2016.05.499

[7] Pagach, D., Warr, R. (2010). The Characteristics of Firms That Hire Chief Risk Officers. Journal of Risk and Insurance, 78 (1), 185-211. doi: https://doi.org/10.1111/j.1539-6975.2010.01378.x

[8] What are the Main Valuation Methods? Available at: https:/corporatefinanceinstitute.com/resources/knowledge/valuation/ valuation-methods/

[9] Ramzaev, M. (2003). Otsenka stoimosti IT-kompaniy: nadezhnye metody est'. Available at: https://www.cnews.ru/articles/ otsenka_stoimosti_itkompanij_nadezhnye

[10] Skarga-Bandurova, I., Derkach, M., Kotsiuba, I. (2018). The Information Service for Delivering Arrival Public Transport Prediction. 2018 IEEE 4th International Symposium on Wireless Systems Within the International Conferences on Intelligent Data Acquisition and Advanced Computing Systems (IDAACS-SWS). doi: https://doi.org/10.1109/idaacs-sws.2018.8525787

[11] Sun, J., Li, H. (2011). Dynamic financial distress prediction using instance selection for the disposal of concept drift. Expert Systems with Applications, 38 (3), 2566-2576. doi: https://doi.org/10.1016/j.eswa.2010.08.046

[12] Muralidharan, R. (2004). A framework for designing strategy content controls. International Journal of Productivity and Performance Management, 53 (7), 590-601. doi: https://doi.org/10.1108/17410400410561213 
[13] ISO/IEC 31010:2009 Risk management - Risk assessment techniques (IDT) (2009). Available at: https://www.iso.org/ standard/51073.html

[14] Henley, E. J., Kumamoto, H. (1981). Reliability Engineering and Risk Assessment. By Englewood Cliffs, Prentice-Hall, New Jersey.

[15] Baldwin, J., Lin, Z. (2002). Impediments to advanced technology adoption for Canadian manufacturers. Research Policy, 31 (1), 1-18. doi: https://doi.org/10.1016/s0048-7333(01)00110-x

[16] Chapman, C., Ward, S. (1996). Project risk management: processes, techniques and insights. John Wiley, 322.

[17] DeMarco, T., Lister, T. (2003). Waltzing with Bears: Managing Risk on Software Projects. Dorset House, 144.

\title{
APPLICATION OF A CONVOLUTIONAL NEURAL NETWORK AND A KOHONEN NETWORK FOR ACCELERATED DETECTION AND RECOGNITION OF OBJECTS IN IMAGES
}

\author{
Victor Skuratov \\ All-Russian Research Institute of Radio Engineering \\ 22 Bol'shaya Pochtovaya str., Moscow, Russian Federation, 105082 \\ viktor.skuratov@gmail.com \\ Konstantin Kuzmin \\ Department of Mathematical and Instrumental Methods in Economics \\ University of Russian Innovation Education \\ 10 Krasnobogatyrskaya str., Moscow, Russia, 107061 \\ konstantin.alexandrovich@yahoo.com \\ Igor Nelin \\ Department of Radiolocation, Radio Navigation and On-Board Radio Electronic Equipment \\ Moscow Aviation Institute \\ 4 Volokolamskoe highway, Moscow, Russian Federation, 125993 \\ nelin.iv@yandex.ru \\ Mikhail Sedankin \\ State Research Center - Burnasyan Federal Medical Biophysical Center of \\ Federal Medical Biological Agency \\ 23 Marshala Novikova str., Moscow, Russia, 123098 \\ Department of Fundamentals of Radio Engineering \\ National Research University "Moscow Power Engineering Institute" \\ 14 Krasnokazarmennaya str., Moscow, Russian Federation, 111250 \\ msedankin@yandex.ru
}

\footnotetext{
Abstract

One of the most effective ways to improve the accuracy and speed of algorithms for searching and recognizing objects in images is to pre-select areas of interest in which it is likely to detect objects of interest. To determine areas of interest in a pre-processed radar or satellite image of the underlying surface, the Kohonen network was used. The found areas of interest are sent to the convolutional neural network, which provides the final detection and recognition of objects. The combination of the above methods allows to speed up the process of searching and recognizing objects in images, which is becoming more expedient due to the constantly growing volume of data for analysis. The process of preliminary processing of input data is described, the process of searching and recognizing patterns of aircraft against the underlying surface is presented, and the analysis of the results is carried out. The use of
} 